\title{
Do men need special services?
}

\author{
Harry Kennedy
}

This is the second paper in this issue belonging to a series of contributions to APT concerning gender and mental health (Bartlett \& Hassell, 2001; Cremona E Etchegoyen, 2001; Kennedy, 2001, this issue; Kohen, 2001b; Kohen E Arnold, 2001; see also Kohen, 2001a, this issue).

The targeting of services to groups with special needs is today commonplace in enlightened public health policies. To list men among the 'minorities' in need of such special help might have the semblance of satire. This air of levity is not really reduced by listing male's shorter life expectancy, higher infant mortality and higher rates of natural and unnatural deaths in all age groups (Drever \& Bunting, 1997; Kelly \& Bunting, 1998).

\section{Epidemiology}

Recent attention has focused on raising public awareness of testicular and prostatic cancers, a simple analogy with the well-established screening programmes for cancers of the female reproductive system. In psychiatry, higher rates in males of childhood autism, Asperger's syndrome, childhood conduct disorder, attention-deficit hyperactivity disorder ADHD), the greater prevalence of drug and alcohol problems, the predominance of men in the prison population and the rising suicide rate in young men all suggest some systematic developmental disadvantage when compared with women. In spite of this, epidemiological surveys of representative community samples, such as the Epidemiologic Catchment Area survey in the USA and the Office of Population Censuses and Surveys survey in Britain, yield a higher prevalence of depression and anxiety disorders in women, while only alcohol and drug problems are more prevalent in men (Meltzer et al, 1995; Tsuang et al, 1995). Severe mental illness appears equal in men and women, although some recent research suggests that schizophrenia may be more common in men than in women (Riecher et al, 1991; Iacono \& Beiser, 1992; Hafner et al, 1993) (Table 1). Is this epidemiological gap between men and women in suicide rates and prevalence rates of common mental illnesses real, or do the commonly used epidemiological screening instruments lack sensitivity for the more common affective and anxiety disorders in men? Alternatively, are epidemiological survey methods too sensitive to relatively minor (less fatal and less handicapping) disorders in women?

Table 1 Office of Population Censuses and

Surveys (OPCS) odds ratios (from Meltzer et al,

1995) and Epidemiological Catchment Area (ECA)

ratios (from sources cited in Tsuang et al, 1995)

\begin{tabular}{|lll|}
\hline & $\begin{array}{l}\text { OPCS } \\
\text { F:M OR } \\
\text { (adjusted) }\end{array}$ & $\begin{array}{l}\text { ECA } \\
\text { Fatio }\end{array}$ \\
\hline & Equal & $1.81: 1$ \\
\hline $\begin{array}{l}\text { Depression } \\
\text { Generalised anxiety disorder }\end{array}$ & $\begin{array}{l}1.31: 1 \\
\text { Panic }\end{array}$ & \\
Mixed anxiety-depression & $1.73: 1$ & $2.0: 1$ \\
$\begin{array}{l}\text { Alcohol dependence } \\
\text { Drug dependence }\end{array}$ & $0.26: 1$ & \\
$\begin{array}{l}\text { Functional psychoses } \\
\text { Bipolar disorder }\end{array}$ & 0.64 & \\
Schizophrenia & Equal & \\
\hline $\begin{array}{l}\text { 1. For 1-week prevalence. } \\
\text { 2. For 1-month prevalence. }\end{array}$ & & $1: 1$ \\
F, female; M, male. & & F<M? \\
\hline
\end{tabular}

Harry Kennedy is Consultant Forensic Psychiatrist at the Central Mental Hospital, Dundrum, Dublin 14, Ireland. He was formerly Clinical Director of the North London Forensic Psychiatry Service, Enfield Community Care Trust. His research interests include the epidemiology of homicide and suicide as related to deprivation and urbanisation, the organisation of forensic mental health services and the psychopathology of anger. 


\section{Services for men}

The segregation of services according to gender, usually duplicating essentially the same services, has a much longer track record than the modern preference for integration, and is still the usual pattern in many countries. Monasteries, prisons, military forces, schools and universities, hospitals and poor-law institutions were traditionally duplicated or reserved for one or other gender, usually males. While the professions have for the most part moved towards integration, and the more enlightened services such as schools and hospitals have also integrated, some institutions remain separate, notably prisons and high secure hospitals. Where separation continues, it may arise from the predominance of male uptake and use, the real risks of integration or simply from a preference for separation as much as from lack of enlightenment.

A second issue relevant to services for men concerns choice, and the accessibility and acceptability of services. Do men have unrecognised preferences that are preventing them from using health services generally, and mental health services in particular?

In order to consider whether men need special services, this paper follows a system for classifying mental health services in a meaningful way. Rather than dividing mental health services for adults into some series of existing institutional structures (hospital, community, day care, etc.), it may be more informative to consider mental health services as a series of processes. A comprehensive mental health service should have the capacity to raise public awareness and find cases and to assess, treat, rehabilitate and either discharge, transfer or provide ongoing support as required. It should have outcome measures that are relevant to the service's aims and objectives. Even this simple functional anatomy of a service neglects public health education, screening and liaison with primary care, drug and alcohol services, sexual health clinics and other agencies such as employers, social services and the criminal justice system.

\section{Raising awareness and finding cases}

Public health education and screening programmes presume that there are clearly defined problems, currently causing disability or suffering, for which a proven set of interventions exists, which will lead to cost-effective health gains, and that these interventions are funded and available. There is already an awareness that recognised disorders, such as drug and alcohol problems and schizophrenia, often go undiagnosed and untreated. There is evidence that mental illness is more likely to be missed in men, particularly in primary care (Redman et al, 1991). Men with severe mental illness, addictions and other affective illnesses are greatly overrepresented in forensic populations when compared with the general population, whether in court diversion schemes, remand and sentenced prison populations, or high and medium secure hospitals. This means that substantial numbers of men are falling through the net of primary care and mental health services, and failing to seek help voluntarily. That they are eventually criminalised owing to the consequences of their illness is evident from the great excess of such diagnoses in prison populations when compared with community surveys (Office for National Statistics, 1998).

It follows that men with severe mental illness really are prone to being criminalised owing to some failure of mental health services. This failure may be at any point in the process of case finding, assessment, treatment and rehabilitation outlined above. Humphries et al (1992) have shown that many first presentations with severe mental illness (particularly males) are via the police or criminal justice system, although often these presentations are handled informally. The rising suicide rate among young men in inner cities at a time when suicide rates for other groups are falling (Drever \& Bunting, 1997) underlines the same apparent failure in early case finding and early intervention. This failure probably arises from a combination of lack of public awareness and stigma, particularly among men, and lack of accessibility or acceptability of the services on offer.

Public awareness can be enhanced and mental disorders de-stigmatised by media campaigns, such as the Royal College of Psychiatrists' recent 'Defeat Depression Campaign', which deliver simple factual messages in forms targeted to gain the attention and acceptance of groups at risk. If the target group is to be young men with schizophrenia, raising awareness and reducing stigma among parents and friends may be as useful as targeting the sufferer.

\section{Accessibility and acceptability}

Primary care and community psychiatry services tend to be provided during the day and near to home: times and places convenient for women and children. Evening clinics, weekend clinics and access to clinics near to places of work rather than home might all enhance accessibility for men. Acceptability is commonly seen as providing same-gender clinicians and clinicians of similar ethnic, cultural or linguistic background. For mental illness, however, there is evidence that men prefer to disclose such symptoms to female general practitioners (GPs) (Boardman, 1987). 
Avoidance of labelling and normalisation may greatly enhance acceptability, but can ultimately lead to the provision of de-skilled, ineffective services. However, many voluntary sector groups, such as Alcoholics Anonymous, appear to have great success in achieving uptake and engagement through a subtle balancing of disease models for public acceptance, while at the same time using peer pressure to persuade users to accept the need for change and personal responsibility for managing their problem. Where services are selectively for men, or for problems which predominantly affect men, an all-male peer group may be very much more acceptable for some, but off-putting for others. Choice is important, but research in this area is lacking.

\section{Assessment services}

Once patients find their way into primary care and psychiatric services, clinicians themselves may need a greater level of awareness of the manifestations of common mental disorders as they present in men (Redman et al, 1991) and of less common disorders that occur mainly in men. It is a commonplace observation that the diagnosis of mania or hypomania may be delayed if the predominant presentation is with irritability and paranoia rather than elation and grandiosity (Dilsaver et al, 1999). There may be other less-wellknown mental disorders or presentations that account for the selective handicapping or premature mortality of men (e.g. Downs \& Gold 1997; Houston et al, 1997). These might account for the apparent 'epidemiological gap' between suicide and psychiatric morbidity rates in men and women. Drugs and alcohol disorders have already been mentioned. Candidates for unrecognised morbidity might include 'atypical' affective disorders such as distorted grief reactions (Lindemann, 1944) and rage attacks (Fava et al, 1990), attachment disorders (Mullen \& Pathe, 1994), paraphilias, and disorders of habit and impulse related to the addictions, for example, pathological gambling, repetitive self-harm and kleptomania (Orford, 1985).

\section{Developmental disorders}

Autism and Asperger's syndrome, specific learning disabilities, childhood conduct disorder and ADHD are all more common in males. There is increasing interest in the developmental pathways or careers that relate these disorders to adult problems, including substance misuse, criminal behaviour and imprisonment (Wessely \& Taylor, 1991).

Little is known of the long-term consequences of physical and sexual abuse and neglect of boys in childhood, since most epidemiological studies have been confined to girls. Most victims of abuse do not go on to become abusers. However, men who are violent to their partners are more likely to have a history of abuse in childhood, along with current depression and heavy alcohol use (Oriel \& Fleming, 1998). Sexual assault on adult males is likely to be underreported and underrecognised, although the psychiatric consequences can be just as severe as in sexually assaulted women (King, 1990).

\section{Distorted grief reactions}

Grief is not included in standard classifications of mental disorders because it is a normal part of emotional experience. Grief reactions can, however, be diagnosed as episodes of depression if they are sufficiently prolonged, manifest typical abnormal symptoms, such as altered sleep patterns and appetites, and are severe enough to be disabling. Recently published consensus criteria for 'traumatic' grief relied on a sample that was $70 \%$ female (Prigerson $e t$ al, 1999), a source of bias that is seldom commented on. The study identified excessive irritability, bitterness or anger among the criterion symptoms, features consistent with Lindemann's (1944) 'distorted' grief reaction. In men, grief reactions owing to losses other than bereavement may go unrecognised, particularly if symptoms include irritability and resentment. The break-up of a relationship, the loss of contact with children, or the loss of status and independence owing to unemployment can all precipitate pathological, distorted or traumatic grief reactions.

Where there has been an ambivalent dependence on the lost partner, or idealisation of the lost child, with a disproportionate sense of entitlement, the form of the bereavement reaction may not be typical of depression. As in post-traumatic stress disorder, symptoms such as persecutory preoccupations, a pervasive sense of resentment and injustice, irritability, ruminations on themes of revenge or vindication may all be prominent. These angry cognitions and affects may be accompanied by other more typical affective symptoms including sleep and appetite disturbances, altered libido, impaired concentration and thoughts of suicide.

\section{Rage attacks}

There is an established psychopharmacological and physiological equivalence between panic attacks and rage attacks (Fava et al, 1990). A set of definitions for fear and anger (see Box 1) is in keeping with these physiological and pharmacological equivalences, at least in defensive rather than offensive roles. Because the switch between flight and fight, fear and anger, or panic and rage is no more than the perception of others as likely victims or victors, males appear more prone 
to anger or rage, and females more prone to fear or panic. Men with rage attacks also commonly give histories of panic attacks and may be more willing to disclose their rages if first asked about panic attacks.

A person suffering from panic disorder and agoraphobia, usually a woman, can generate a powerful secondary family structure in which she occupies a position of great power through her dependency. This becomes an important continuing factor for her agoraphobia long after panic attacks have become infrequent. Much the same happens in the families of men with rage attacks. The man need seldom repeat a rage attack to command a secondary family structure in which his minor domestic demands are promptly met. At the same time, his reputation as a hard man, a champion in his neighbourhood - an honour not to be disrespected is a further reward that is difficult to relinquish.

Both panic and rage are commonly comorbid with dependence on alcohol, cannabis or benzodiazepines. These give short-term relief of autonomic symptoms, but with chronic use, tolerance may exacerbate the frequency of attacks, probably by lowering the threshold for autonomic reactions and for perception of threat. Substance misuse, by association with rage and panic, acquires in most cultures a double-standard role, a badge of male prestige and female shame.

\section{Attachment disorders}

The pathologies of passion (Mullen \& Pathe, 1994) all appear to be more common in men. Stalking (persistent unwanted attention) has recently been defined as an imprisonable offence in many jurisdictions. The underlying psychopathology can include disorders of courtship (including de Cleramboult's syndrome), abnormalities of attachment (including morbid jealousy; Mullen \& Pathe, 1994) and separation disorders (see Distorted grief above).

\section{Box 1 Anger and fear}

"Anger is an affective state experienced as the motivation to warn, intimidate or attack (or for fear, to escape, appease or avoid) those who are perceived as challenging or threatening. Anger (like fear) is coupled to and is inseparable from a sensitivity to the perception of challenges or a heightened awareness of threats (irritability or anxiety). This affective motivation and sensitivity can be subjectively experienced even if no external action occurs." Kennedy (1992)
The definitions of fear and anger given in Box 1 emphasise rage and panic and probably give insufficient emphasis to the prolonged, pervasive or preoccupying feelings of resentment and humiliation, with a bias towards self-justification and the motivation for revenge or retribution, that characterise a class of predominantly male patients found among stalkers (Mullen \& Pathe, 1994) and male patients with delusional disorder (Kennedy et al, 1992).

\section{Paraphilias}

Although there are no reliable population-based epidemiological studies of the prevalence of paraphilias, all surveys indicate that they are much more common in men (e.g. Gosselin \& Wilson, 1980). Paraphilias do not equate with psychiatric disorder. Sexual offending, however, is commonly the route into psychiatric services. For the clinician, it is important to realise that the natural history of paraphilias has been extensively revised in the light of research in recent years (Abel et al,1988). Multiple paraphilias, with a progression serially from one to another, and increasing risk of offending with certain paraphilias, fantasies and cognitive distortions, are all relevant to assessment and treatment. Evidence for victim-offender cycles in general is surprisingly complex and may differ for males and females (Widom \& White, 1997). For all these reasons, treatments based on addiction models for recovery and relapse prevention appear best supported by published research.

\section{Disorders of habit and impulse}

The natural history of the various substance dependence disorders includes the frequent comorbidity or progression of patients from one such addiction to another. The continuity between chemical dependence disorders and a range of other disorders, such as pathological gambling, bulimia nervosa, repetitive selfharm, kleptomania, hypersexuality and repetitive arson, is understandable, and seems meaningful (Lacey \& Evans, 1986). Orford (1985) has postulated a single class of appetitive disorders with some common underlying cause, whether it is a neurochemical disorder or psychopathological dysfunction of cognition. In fact, it is difficult to identify a distinct population with a statistically abnormal association of multiple disorders (Kennedy \& Grubin, 1990). The advantage of this extended appetite/addictions model is that it lends itself so well to the clearest thinking about responsibility in the forensic setting and to the most effective approaches to relapse prevention.

It is interesting that many such problems seem to be more common in men. Repetitive self-harm, often thought to be typical of women in prison, occurs in a greater number of male prisoners, simply because there 
are so many more men in prison (Gunn et al, 1991). These disorders are often overlooked when taking a psychiatric history. If other disorders of appetites or impulse are sought when assessing men presenting with any one addiction, a clearer understanding of the patient's lifetime development and handicaps is likely to emerge, with an enhanced rapport as a result.

Finally, the description of emotional, substance misuse and dispositional differences that may form clusters, syndromes or career patterns (Toch \& Adams, 1989 ) is still at a very early stage of development.

\section{Treatment}

Treatment for men, as distinct from generic treatment, would consist of specialist treatments for the disorders specific to or more common in men, and settings or institutions specifically for men. While issues of access and acceptability have been dealt with above, it is worth considering that, at present, medium and high secure institutions are overwhelmingly for men, usually $85 \%$ or more. This is not new. The three hospitals study (Wing \& Brown, 1961), which first identified the impoverished therapeutic milieu and iatrogenic side-effects of treatment in the underresourced mental hospitals of the 1950s, studied only female patients because there were so few of them in the hospitals studied. It could be argued that one of the impoverishments and artificialities of the therapeutic milieu was the single-gender environment itself.

Wards in modern medium secure units have been mixed since their inception, although it can be difficult to sustain a critical mass of female patients to provide women with a peer group. Where secure psychiatric wards are single-gender, almost entirely in the special hospitals, both male and female single-gender wards are pervaded by a culture of pecking-order disputes, factionalism and competition among patients for the most dramatic of gestures resembling descriptions of prison culture (Toch, 1972). It would be unfair to make the obvious adverse comparison with mixed-gender acute wards and medium-secure units, which do not admit the most extremely aggressive and have closer, more normalising contact with the community. Mixed wards have been criticised for the victimisation of female patients (Barlow \& Wolfson, 1997). Although objective comparisons with single-gender wards (male or female) are difficult to find, mixed wards are undoubtedly more dangerous for women. Mixed environments are, however, probably better therapeutic environments for men and for women, and probably only a few men need to be confined to an all-male environment because of a tendency towards sexual predation. The failure of some institutions to ensure that mixed environments are safe for women (or men) represents a failure of relational and procedural security, almost always owing to poor resource management and lack of regard for quality of care.

\section{Rehabilitation}

It is difficult to conceive of a rehabilitation need for men that would be distinct from a generic, mixedgender service. The need for meaningful work, camaraderie and social intercourse is now integrated for all parts of society. There may, none the less, be substantial immigrant and ethnic minority groups, particularly in the larger cities, for whom the accustomed peer group is by choice same-gender. A tension may exist in acceding to this choice in some cases. Much of the morbidity specific to men may relate to dysfunctional beliefs and attitudes towards women. Treatment, which may have started in all-male peergroup settings, if it is to be consolidated, is likely to require exposure and confrontation in mixed company, to bring about change in practice.

\section{Continuing care}

The greatest failing of community care has been identified as failure to provide community alternatives for the new long-stay patients with severe mental illness. These are predominantly young men. Among their most common sources of dissatisfaction is their difficulty in finding long-term partners and, with this, a common refusal to adhere to prescribed medication, often on the grounds that it causes impotence. Such complaints are seldom followed by careful, tactful enquiry as to the nature of the impotence: erectile failure, failure of ejaculation or any of the other problems, such as premature ejaculation or simple loss of confidence, may or may not be present. Just as important may be the loss of assertiveness and energy owing to oversedation. There is no easy solution to such dissatisfactions, which ultimately arise from the handicaps of the most devastating of diseases. Nevertheless, a greater awareness of the complaints and beliefs of men with severe mental illness might allow more successful rapport between the patient and his clinician.

\section{Conclusions}

Thinking about groups not normally considered as separable can identify new approaches to older problems. The preponderance of Black patients in secure 
units and detained under the Mental Health Act at every level of psychiatric care is largely a preponderance of Black men. Making primary care and local psychiatric services accessible and acceptable to men in general may ameliorate at least a part of this older and more worrying problem. Psychiatric treatment is often perceived as a misuse of power and authority for social control rather than for health. A greater understanding of the importance of dominance and hierarchies in mental illness and psychiatric institutions generally, particularly where male patients are in the majority, is essential to the maintenance of an effective, safe and humane therapeutic environment.

In future, identifying unmet needs in communities will only start with epidemiological studies of morbidity. Mental health services will probably need the services of advertising agencies, with their skills in identifying needs using qualitative methods for specific groups. The preferences, prejudices and access needs of men, once discovered, will then need to be addressed through advertising campaigns, staff selection and training, and flexible provision of services in time and place.

Rather than devise outcome measures for men's services, it would be more immediately productive to incorporate into existing performance measures a greater awareness of the need to demonstrate that services are being used by specific target groups. Existing mental health services probably do fall short of minimum standards for reaching men, just as they fail to reach many large minority groups.

\section{References}

Abel, G. G., Becker, J. V., Cunningham-Rathner, J., et al (1988) Multiple paraphilic diagnoses among sex offenders. Bulletin of the American Academy of Psychiatry and Law, 16, 153-168.

Barlow, F. \& Wolfson, P. (1997) Safety and security: a survey of female psychiatric in-patients. Psychiatric Bulletin, 21, 270-272.

Bartlett, A. \& Hassell, V. (2001) Do women need special services? Advances in Psychiatric Treatment, in press.

Boardman, A. P. (1987) The General Health Questionnaire and the detection of emotional disorder by general practitioners. A replicated study. British Journal of Psychiatry, 151, 373-381

Cremona, A. \& Etchegoyen, A. (2001) Part-time training and training for male and female psychiatrists. Advances in Psychiatric Treatment, in press.

Dilsaver, S. C., Chen, Y. R., Shoaib, A. M., et al (1999) Phenomenology of mania: evidence for distinct depressed, dysphoric and euphoric presentations. American Journal of Psychiatry, 156, 426-430.

Downs, K. \& Gold, S. R. (1997) The role of blame, distress and anger in the hypermasculine man. Violence and Victims, 12, 19-35.

Drever, F. \& Bunting, J. (1997) Patterns and trends in male mortality. In Health Inequalities (eds F. Drever \& M. Whitehead). London: Stationery Office.

Fava, G. A., Anderson, K. \& Rosenbaum, J. F. (1990) “Anger attacks": possible variants of panic and major depressive disorders. American Journal of Psychiatry, 147, 867-870.

Gosselin, C. \& Wilson, G. (1980) Sexual Variations: Fetishism Transvestism and Sado-Masochism. London: Faber \& Faber

Gunn, J., Maden, A. \& Swinton, M. (1991) Treatment needs of prisoners with psychiatric disorders. British Medical Journal, 303, 338-341.
Hafner, H., Maurer, K. \& Riecher-Rossler, A. (1993) The influence of age and sex on the onset and early course of schizophrenia. British Journal of Psychiatry, 162, 80-86.

Houston, B. K., Babyak, M. A., Chesney, M. A., et al (1997) Social dominance and 22-year all-cause mortality in men. Psychosomatic Medicine, 59, 5-12.

Humphries, M. S., Johnstone, E. C., MacMillan, J. F., et al (1992) Dangerous behaviour preceding first admissions for schizophrenia. British Journal of Psychiatry, 161, 501-505.

Iacono, W. \& Beiser, M. (1992) Where are the women in first-episode studies of schizophrenia? Schizophrenia Bulletin, 18, 471-480.

Kelly, S. \& Bunting, J. (1998) Trends in suicide in England and Wales, 1982-96. Population Trends, 92, 29-41.

Kennedy, H. G. (1992) Anger and irritability. British Journal of Psychiatry, 161, 145-153.

— \& Grubin, D. H. (1990) Hotheaded or impulsive? British Journal of Addictions, 85, 639-643.

-, Kemp, L. I. \& Dyer, D. E. (1992) Fear and anger in delusional (paranoid) disorder: the association with violence. British Journal of Psychiatry, 160, 488-492.

King, M. B. (1990) Male rape. British Medical Journal, 302, 179.

Kohen, D. (2001a) Gender and mental health: recognition of unresolved issues. Advances in Psychiatric Treatment, 7, 3-4.

- $(2001 b)$ Psychiatric services for women. Advances in Psychiatric Treatment, in press.

- \& Arnold, E. (2001) Professional, personal, social issues in the life of the femal psychiatrists. Advances in Psychiatric Treatment (in press).

Lacey, L. H. \& Evans, C. H. (1986) The impulsivist: a multiimpulsive personality disorder. British Journal of Addictions, $81,641-649$

Lindemann, E. (1944) Symptomatology and management of acute grief. American Journal of Psychiatry, 101, 141-148.

Meltzer, H., Gill, B., Petticrew, M., et al (1995) The Prevalence of Psychiatric Morbidity among Adults Living in Private Households. OPCS Surveys of Psychiatric Morbidity in Great Britain, Report 1, pp. 66-94. London: HMSO.

Mullen, P. E. \& Pathe, M. (1994) The pathological extensions of love. British Journal of Psychiatry, 165, 614-623.

Office for National Statistics (1998) Psychiatric Morbidity among Prisoners in England and Wales. London: HMSO.

Orford, J. (1985) Excessive Appetites: A Psychological View of Addictions. Chichester: John Wiley \& Sons.

Oriel, K. A. \& Fleming, M. F. (1998) Screening men for partner violence in a primary care setting. A new strategy for detecting domestic violence. Journal of Family Practice, 46, 493-498.

Prigerson, H. G., Shear, M. K., Jacobs, S. C., et al (1999) Consensus criteria for traumatic grief. A preliminary empirical test. British Journal of Psychiatry, 174, 67-73.

Ramsay, R., Welch, S. \& Youard, E. (2001) Needs of women patients with mental illness. Advances in Psychiatric Treatment, 7, 85-92.

Redman, S., Webb, G. R., Hennrikus, D. J., et al (1991) The effects of gender on diagnosis of psychological disturbance. Journal of Behavioural Medicine, 14, 527-540.

Riecher, A., Maurer, K., Loffler, W., et al (1991) Gender differences in age at onset and course of schizophrenic disorders. In Search for the Causes of Schizophrenia (eds H. Hafner \& W. F. Gattaz), pp. 14-33. Berlin: SpringerVerlag.

Toch, H. (1972) Violent Men: An Inquiry into the Psychology of Violence. Harmondsworth: Penguin.

— \& Adams, K. (1989) The Disturbed Violent Offender. New Haven: Yale University Press.

Tsuang, M. T., Tohen, M. \& Zahner, E. P. (eds) (1995) Textbook in Psychiatric Epidemiology, pp. 317-344. New York: WileyLiss.

Wessely, S. \& Taylor, P. J. (1991) Madness and crime: criminology versus psychiatry. Criminal Behaviour and Mental Health, 1, 193-228.

Widom, C. S. \& White, H. R. (1997) Problem behaviours in abused and neglected children grown up: prevalence and co-occurrence of substance abuse, crime and violence. Criminal Behaviour and Mental Health, 7, 287-310.

Wing, J. K. \& Brown, G. W. (1961) Social treatment of chronic schizophrenia: a comparative survey of three mental hospitals. Journal of Mental Science, 107, 847-861. 


\section{Multiple choice questions}

1. Services for men are more accessible and acceptable if:

a public education targets men and men's problems

b primary care and mental health clinics are provided at times and places appropriate for families

c media campaigns are aimed at family and friends as well as the patient at risk

d criminal justice agencies raise awareness and know how to divert to psychiatric services

e they offer the choice of a male clinician.

2. Mental disorders and problems more common in men include:

a specific learning disabilities

b deaths by suicide and homicide

c gambling, self-harm and substance misuse

d courtship and attachment disorders

e rage attacks and chronic resentments.

3. Treatment and rehabilitation services for men should:

a focus on addictions as primary or comorbid problems

b recognise psychosexual difficulties as comorbid problems c allow patients to sort out bullying and peckingorder behaviour

$\mathrm{d}$ address issues of violence and coercion in the family and workplace

e recognise grief and attachment issues regarding loss of contact with children and others.

4. Service developments should:

a ensure a balance between single-gender and mixed treatment settings

$b$ be based on local preferences and offer appropriate choice

c be blind to race, culture, ethnicity and gender

$\mathrm{d}$ raise awareness of less-common disorders that occur predominantly in men

e emphasise the similarities between presentations of mental illness in men and women.

\begin{tabular}{llll}
\multicolumn{5}{l}{ MCQ answers } & & \\
& & & \\
1 & 2 & 3 & 4 \\
a T & a F & a T & a T \\
b F & b T & b T & b T \\
c T & c T & c F & c F \\
d T & d T & d T & d T \\
e T & e F & e T & e F
\end{tabular}

\section{Commentary}

\section{Jennie Williams}

The papers by Ramsay et al and Kennedy in this issue take us on a journey over the bumpy terrain of gender differences in mental health and mental health service provision. My intention is not to comment on the finer points of their journeys, rather to suggest that they would have concluded more satisfactorily if greater reference had been made to the existence of social inequalities.

\section{Social inequalities and mental health}

There is ample evidence (e.g. Harris \& Landis, 1997; Newnes et al, 1999; Milne \& Williams, 2000) that

Jennie Williams is a clinical pyschologist with a background in social psychology. She is senior lecturer in mental health at the Tizard Centre (University of Kent at Canterbury, Canterbury, Kent CT2 7LZ; tel: 01227 824047; fax: 01227 763674; e-mail: J.A.Williams@tizard.ukc.ac.uk). Her main research interest is the effects of social inequalities on mental health and service provision. 